\title{
Genome-scale Arabidopsis promoter array identifies targets of the histone acetyltransferase GCN5
}

\author{
Moussa Benhamed ${ }^{1}$, Marie-Laure Martin-Magniette ${ }^{2,3}$, Ludivine Taconnat ${ }^{2}$, Frédérique Bitton ${ }^{2}$, Caroline Servet ${ }^{1}$, \\ Rebecca De Clercq ${ }^{4,5}$, Björn De Meyer ${ }^{4,5}$, Caroline Buysschaert ${ }^{4,5}$, Stéphane Rombauts ${ }^{4,5}$, Raimundo Villarroel ${ }^{4,5}$, \\ Sébastien Aubourg ${ }^{2}$, Jim Beynon ${ }^{6}$, Rishikesh P. Bhalerao ${ }^{7}$, George Coupland ${ }^{8}$, Wilhelm Gruissem ${ }^{9}$, Frank L.H. Menke ${ }^{10}$, \\ Bernd Weisshaar ${ }^{11}$, Jean-Pierre Renou ${ }^{2}$, Dao-Xiu Zhou ${ }^{1, *}$ and Pierre Hilson ${ }^{4,5}{ }^{*}$ \\ ${ }^{1}$ Institut de Biotechnologie des Plantes, UMR 8618, Centre National de la Recherche Scientifique, Université de Paris Sud 11, \\ 91405 Orsay, France, \\ ${ }^{2}$ Unité de Recherche en Génomique Végétale, UMR INRA 1165-CNRS 8114-UEVE, 91057 Evry Cedex, France, \\ ${ }^{3}$ Unité de Mathématiques et Informatique Appliquées, UMR AgroParisTech-INRA, 75231 Paris Cedex, France, \\ ${ }^{4}$ Department of Plant Systems Biology, Flanders Institute for Biotechnology, 9052 Gent, Belgium, \\ ${ }^{5}$ Department of Molecular Genetics, Ghent University, 9052 Gent, Belgium, \\ ${ }^{6}$ Warwick Horticultural Research International, University of Warwick, Warwick CV35 9EF, UK, \\ ${ }^{7}$ Department of Forest Genetics and Plant Physiology, Swedish University of Agricultural Sciences, S-901 83 Umeå, Sweden, \\ ${ }^{8}$ Abteilung Entwicklungsbiologie der Pflanzen, Max-Planck-Institut für Züchtungsforschung, 50829 Köln, Germany, \\ ${ }^{9}$ Institute of Plant Sciences, Eidgenössische Technische Hochschule Zurich, 8092 Zurich, Switzerland, \\ ${ }^{10}$ Molecular Genetics Group, Department of Biology, Utrecht University, 3584 CH Utrecht, The Netherlands, and \\ ${ }^{11}$ Department of Biology, Genome Research, Bielefeld University, 33594 Bielefeld, Germany
}

Received 6 April 2008; revised 6 June 2008; accepted 10 June 2008; published online 15 August 2008.

*For correspondence (fax +32 9331 3809; e-mail pierre.hilson@psb.ugent.be or fax +33 16915 6103; e-mail dao-xiu.zhou@u-psud.fr).

\begin{abstract}
Summary
We have assembled approximately 20000 Arabidopsis thaliana promoter regions, compatible with functional studies that require cloning and with microarray applications. The promoter fragments can be captured as modular entry clones (MultiSite Gateway format) via site-specific recombinational cloning, and transferred into vectors of choice to investigate transcriptional networks. The fragments can also be amplified by PCR and printed on glass arrays. In combination with immunoprecipitation of protein-DNA complexes (ChIP-chip), these arrays enable characterization of binding sites for chromatin-associated proteins or the extent of chromatin modifications at genome scale. The Arabidopsis histone acetyltransferase GCN5 associated with $40 \%$ of the tested promoters. At most sites, binding did not depend on the integrity of the GCN5 bromodomain. However, the presence of the bromodomain was necessary for binding to $11 \%$ of the promoter regions, and correlated with acetylation of lysine $\mathbf{1 4}$ of histone $\mathrm{H} 3$ in these promoters. Combined analysis of ChIP-chip and transcriptomic data indicated that binding of GCN5 does not strictly correlate with gene activation. GCN5 has previously been shown to be required for light-regulated gene expression and growth, and we found that GCN5 targets were enriched in early light-responsive genes. Thus, in addition to its transcriptional activation function, GCN5 may play an important role in priming activation of inducible genes under non-induced conditions.
\end{abstract}

Keywords: Arabidopsis, chromatin immunoprecipitation, histone acetylation, ChIP-chip, promoter, Gateway site-specific recombination.

Introduction

By affecting nucleosome positioning and density, histone modifications play a pivotal role in chromatin remodeling and gene regulation (Horn and Peterson, 2002). Covalent additions to the $\mathrm{N}$-terminal tails of the core histones include 
acetylation, methylation, phosphorylation, ADP ribosylation and ubiquitinylation. These modifications dynamically shape chromatin structure in various, sometimes opposite, ways. Their pattern determines the open or repressed state of the cognate DNA sequences (Jenuwein and Allis, 2001). In particular, hyper-acetylation relaxes chromatin structure and is associated with transcriptional activation, whereas hypo-acetylation induces chromatin compaction and gene repression (Millar and Grunstein, 2006). Histone acetylation also provides epigenetic markers for gene expression because it blocks association of heterochromatin-stabilizing complexes.

The homeostatic balance of nucleosomal histone acetylation is maintained by the antagonistic action of histone acetyltransferases (HATs) and histone deacetylases (HDACs). HAT and HDAC proteins usually function in multi-protein complexes. For instance, the yeast HAT protein Gcn5 is the catalytic subunit of the Spt-Ada-Gcn5 acetyltransferase (SAGA), SAGA-like (SLIK), transcriptional adaptator (ADA) and HAT-A2 complexes that have distinct histone targets and effects on transcriptional activity (reviewed in Lee and Workman, 2007). In addition to its role in histone acetylation, Gcn5 primarily functions as a key subunit of transcriptional co-activator complexes.

Chromatin regulators, including HAT complexes, have no sequence-specific DNA recognition properties of their own and are recruited to specific locations in the genome by various mechanisms (Lee and Workman, 2007). First, GCN5 association with promoters can be mediated directly or indirectly by DNA-binding transcription factors (Robert et al., 2004). For example, in yeast, Gcn5 association with the $\mathrm{HO}$ promoter depends on the transcription factor SBF (Cosma et al., 1999). In other cases, GCN5 is bound to promoters through interaction between GCN5-containing complexes, such as ADA or SAGA, and promoter-binding transcription factors. In Arabidopsis thaliana, the coldresponsive transcription factor CBF1 was recently shown to interact with ADA2, which in turn binds GCN5 (Mao et al., 2006). Second, the GCN5-containing SAGA complex can be recruited to promoters by recognizing specific histone markers, such as the trimethylated $\mathrm{H} 3 \mathrm{~K} 14$ residue (Pray-Grant et al., 2005). Third, the GCN5 bromodomain binds to acetylated histone tails, thereby mediating GCN5 association with chromatin for propagation of general histone acetylation (Imoberdorf et al., 2006; Mujtaba et al., 2007).

The Arabidopsis HAT family contains 12 members, which fall into four groups based on primary homology with yeast and mammalian HATs: GCN5-related N-acetyltransferase (GNAT), MOZ-YBF2/SAS3-SAS2-TIP60 (MYST), CREBBinding Protein (CBP) and TBP-associated factor 1 (TAF1) (Pandey et al., 2002). Among the GNAT members, GCN5 (or HAG1) has been functionally characterized. GCN5 is evolutionarily conserved, with orthologs in other eukaryotes, and contains a HAT N-terminal catalytic domain and a C-terminal bromodomain (Pandey et al., 2002). Deletion mutations of GCN5 result in either up or downregulation of $4 \%$ of the genes in yeast and cause embryo growth defects in mice (Lee et al., 2000; Yamauchi et al., 2000). In Drosophila melanogaster, null $d G c n 5$ alleles block the onset of both oogenesis and metamorphosis (Carré et al., 2005). T-DNA insertion mutations of Arabidopsis GCN5 induce pleiotropic defects that alter plant development and responses to environmental conditions, such as light and cold (Benhamed et al., 2006; Bertrand et al., 2003; Vlachonasios et al., 2003), suggesting that GCN5 is involved in both the long-term regulation of epigenetic chromatin landscapes and the control of short-term transcriptional switches. Mutations in GCN5 affect the expression of approximately $4 \%$ of the Arabidopsis genes in seedlings (Vlachonasios et al., 2003). GCN5 is involved in the acetylation of both histone $\mathrm{H} 3$ and $\mathrm{H} 4$ near the proximal promoter region of several light-inducible genes, suggesting that it is recruited by DNA-binding transcription factors (Benhamed et al., 2006).

Although GCN5 plays an essential role in chromatin modification and transcriptional regulation, its mode of action is still not understood. Therefore, it is important to determine which sites are targeted by GCN5 in the Arabidopsis genome in order to investigate the mechanisms it regulates. In recent years, whole-genome array technologies have been used to identify the sites bound by chromatinassociated proteins in vivo (Hanlon and Lieb, 2004) or targeted by chromatin-modifying enzymes (Millar and Grunstein, 2006; Zhang et al., 2006). We used chromatin immunoprecipitation (ChIP) in combination with a novel Arabidopsis promoter array to demonstrate that GCN5 associates with a large number of transcriptionally active promoters. GCN5-promoter binding appeared to be mostly independent of the bromodomain that recognizes acetylated histone tails (reviewed in Mujtaba et al., 2007). We found, however, that the bromodomain is required for binding and acetylating lysine 14 of histone $\mathrm{H} 3$ on a subset of promoter regions, $10 \%$ of which are promoters of transcription factor genes. Considering light-regulated genes, GCN5 target promoters are enriched in early lightresponsive genes.

\section{Results}

\section{The Arabidopsis SAP promoter repertoire}

To facilitate the study of transcriptional networks in Arabidopsis, we developed a reference repertoire of Arabidopsis promoter sequences compatible with both microarray applications and downstream functional studies that require cloning (Figure 1). The systematic analysis of Arabidopsis promoters (SAP) repertoire was constructed 


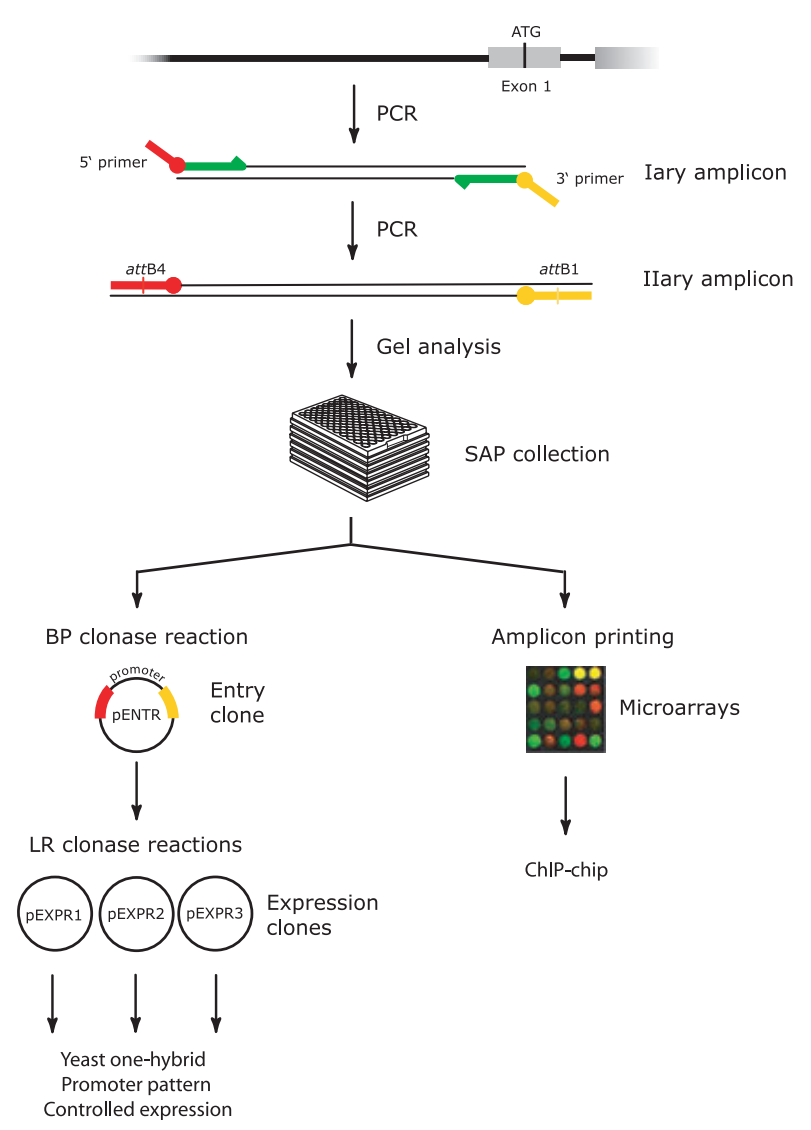

Figure 1. Construction and exploitation of the SAP promoter repertoire.

in two successive rounds. The first phase focused on gene models supported by experimental full-length cDNA sequences available in GenBank (December 2003), and included an in silico set of 11893 promoter amplicons (SAPv1). The second phase extended the repertoire to all gene models whose transcription could be detected in the AtGenExpress ATH1 genechip profile compendium (http://www.arabidopsis.org/info/expression/ATGenExpress. jsp), complemented by data obtained by Yamada et al. (2003), resulting in the addition of 10367 promoter amplicons, giving a total set of 22260 in silico amplicons (SAPv2).

Each promoter amplicon was defined in silico by a pair of oligonucleotides designed for PCR synthesis from genomic DNA of the Arabidopsis Columbia-0 ecotype. The amplicon $3^{\prime}$ end was the nucleotide preceding the translation initiation codon in the corresponding gene model. Because multiple transcription initiation sites can be defined for individual genes, the amplified fragments also included the corresponding $5^{\prime}$ untranslated region, although here they are referred to as promoters. The amplicon $5^{\prime}$ end was located at the boundary between the targeted intergenic region and the adjacent upstream open reading frame
(ORF), or approximately $2.0 \mathrm{~kb}$ upstream of the translation initiation codon.

\section{SAP database}

Promoter amplicon information is available via the SAP online database at http://www.psb.ugent.be/SAP/. For each amplicon, the database provides its sequence (as deduced from the Arabidopsis genome), PCR primers, length, genome location, percentage GC content, corresponding gene code, and additional technical information. The relevant information can also be downloaded as a tab-delimited dump file (http://www.psb.ugent.be/SAP/). The SAP promoter repertoire has been integrated into the FLAGdb++ genome viewer (http://urgv.evry.inra.fr/FLAGdb; Samson et al., 2004), in which details about individual sequences can be accessed by clicking on their graphical representation.

\section{Promoter amplicon synthesis}

In total, 19741 promoter amplicons were successfully synthesized, as verified by the detection of a single PCR product of the expected size by agarose gel electrophoresis. Most other PCRs did not yield a product that was detectable in the gel because of the amplicon size. Sequences of $2.0 \mathrm{~kb}$ or smaller are preferred, because larger fragments yielded less robust PCR amplifications. All primary amplification results are documented in the SAP database, including links to agarose gel images and 96-well microtiter plate maps with PCR results. Promoter sequences were amplified in a two-step PCR for addition of universal extensions carrying the attB1 and attB4 Gateway cloning sites (Figure 1).

During the first phase of the SAP project, the SAPv1 collection (consisting of 124 96-well plates) was re-amplified in a subsequent PCR. Agarose gel analysis of PCR products confirmed the synthesis of 8471 probes of the expected size and in sufficient amount. Below, we present two SAPv1 array applications for studying the association between chromatin and remodeling enzymes and the acetylation status of core histones.

\section{Genome-wide association of GCN5 with target promoters}

Arabidopsis promoters bound by GCN5 in vivo were identified in ChIP-chip assays. A purified polyclonal antibody used to immunoprecipitate chromatin fragments was raised against two GCN5 peptides located in the N-terminal portion of the protein (Benhamed et al., 2006). The antibody detected a protein of $62 \mathrm{kDa}$ in the wild-type, as expected for the GCN5 protein, but smaller polypeptides in the gcn5-1 and gcn5-2 mutants that each carry a T-DNA insertion in the $3^{\prime}$ end of the gene (Figure S1). The T-DNA insertion in gcn5-1 


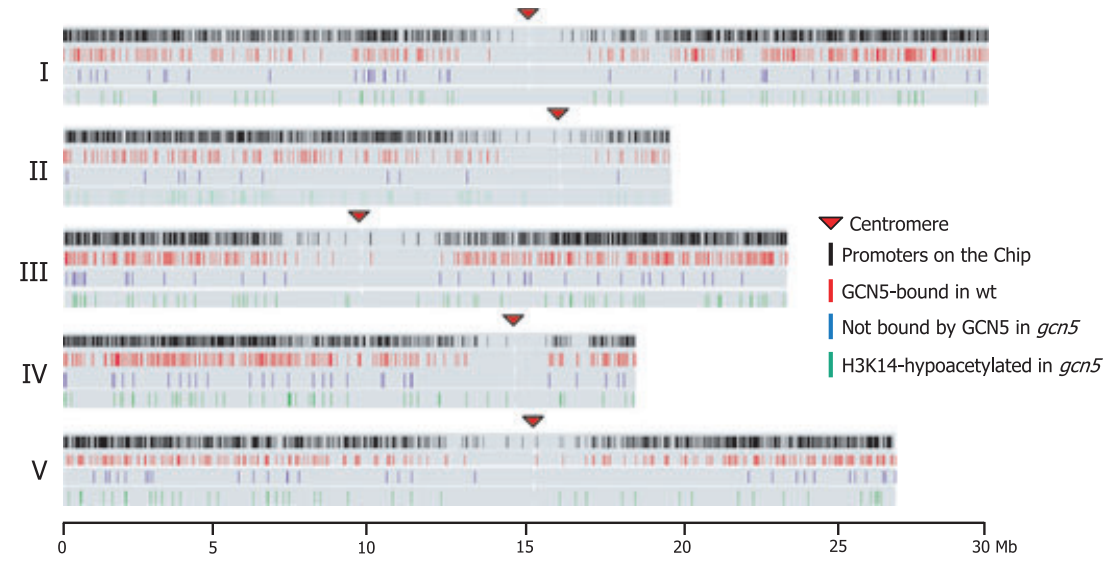

Figure 2. Genome-wide distribution of Arabidopsis GCN5 targets. From top to bottom and for each Arabidopsis promoter (I-V): red arrowheads, centromeres; black, position of promoter sequences spotted on the SAPv1 array; red, GCN5 binding promoter targets; blue, promoters for which GCN5 binding was abolished in the gcn5-1 and gcn5-2 mutants; green, promoters that were H3K14 hypo-acetylated in the gcn5-1 and gcn5-2 mutants. removes 17 codons of the C-terminal bromodomain (Vlachonasios et al., 2003), whereas the insertion in gcn5-2 results in complete deletion of the bromodomain (Bertrand et al., 2003; Long et al., 2006).

GCN5-associated chromatin fractions were extracted from wild-type, gcn5-1 and gcn5-2 plants, and three biological ChIP-chip replicates were analyzed for each of them. The DNA purified in each ChIP (defined as the IP fraction) was amplified, labeled, and hybridized to the promoter array. After data normalization to remove technical biases, the IP hybridization signal histogram was modeled using a mixture model: components of the mixture were interpreted to identify 'enriched' probes corresponding to GCN5-associated promoter regions (see Experimental procedures).

ChIP-chip results showed that, in wild-type plants, GCN5 binds to a large proportion of the promoters contained in the SAPv1 promoter repertoire (3352/8471, i.e. $40 \%$; Table S1). Target promoters were distributed over all five chromosomes (Figure 2). Chromatin isolated from gcn5-1 and gcn5-2 plants had comparable profiles (Pearson correlation $=0.78$, calculated between the ratio of the log-ratio (IP/input) of gcn5-1 over the log-ratio (IP/input) of wild type and the ratio of the log-ratio (IP/input) of gcn5-2 over the log-ratio (IP/ input) of wild type). Comparative analysis revealed that $89 \%$ (2975/3352) of the GCN5 target promoters bound in wild-type plants were precipitated from the chromatin of both mutants, indicating that the truncated GCN5 proteins were still recruited to a large proportion of the target promoters (Figure $3 \mathrm{~b}$ and Table S1). This result implies that the remaining promoters that were targeted in the wild-type, but not in the mutants, require the GCN5 bromodomain for binding (Figures 2 and $3 b$ ).

To assess the reliability of the ChIP-chip results, we used conventional ChIP-PCR to test the enrichment of seven promoters randomly selected from those targeted in the wild-type but not in the gcn 5 mutants. All tested promoters were confirmed to be enriched in the wild-type samples by this independent assay (Figure $3 c$ and Table S2).
Requirement of the GCN5 bromodomain for chromatin binding and acetylation of lysine 14 of histone $\mathrm{H} 3$

We have previously shown that the gcn5-2 mutation results in a clear decrease of histone $\mathrm{H} 3$ acetylation in nucleosomes located in the vicinity of the core promoter regions of three light-regulated genes (namely $C A B 2, R B C S-1 A$ and $I A A$ ), and that GCN5 is essential for acetylation of lysine 14 of histone H3 (H3K14; Benhamed et al., 2006). We hypothesized that the bromodomain of GCN5 protein was important for acetylation of $\mathrm{H} 3 \mathrm{~K} 14$.

To investigate the role of the GCN5 bromodomain in H3K14 acetylation at the genome scale, chromatin of gcn5-1, gcn5-2 and wild-type plants was immunoprecipitated with an antibody that specifically recognizes acetylated H3K14. Our analysis revealed 564 promoters in the mutants for which H3K14 was hypo-acetylated compared to wild-type (Figure 3b). All hypo-acetylated promoters were among the GCN5 binding targets in wild-type plants, indicating a clear functional link between GCN5 binding and H3K14 acetylation. Of these promoters, 215 (38\%) were still bound by GCN5 in the mutants. The remaining $349(62 \%)$ promoters corresponded to sites that were no longer bound by the mutant GCN5 proteins, representing 93\% (349/377) of the target promoters that required the bromodomain for GCN5 binding. These data suggest that the GCN5 bromodomain is necessary to acetylate $\mathrm{H} 3 \mathrm{~K} 14$ in a subset of promoter regions. In some cases, lack of acetylation could reflect the inability of the mutant GCN5 protein to bind chromatin. In other cases, the mutant protein was associated with chromatin but unable to acetylate H3K14.

\section{Functional classification of GCN5 targets}

Classification of GCN5 target promoters based on gene ontology showed that GCN5 bound to a wide range of promoters of genes with diverse functions (Figure 4), and that GCN5 binding was not obviously biased for any particular gene ontology category. However, promoters bound to 

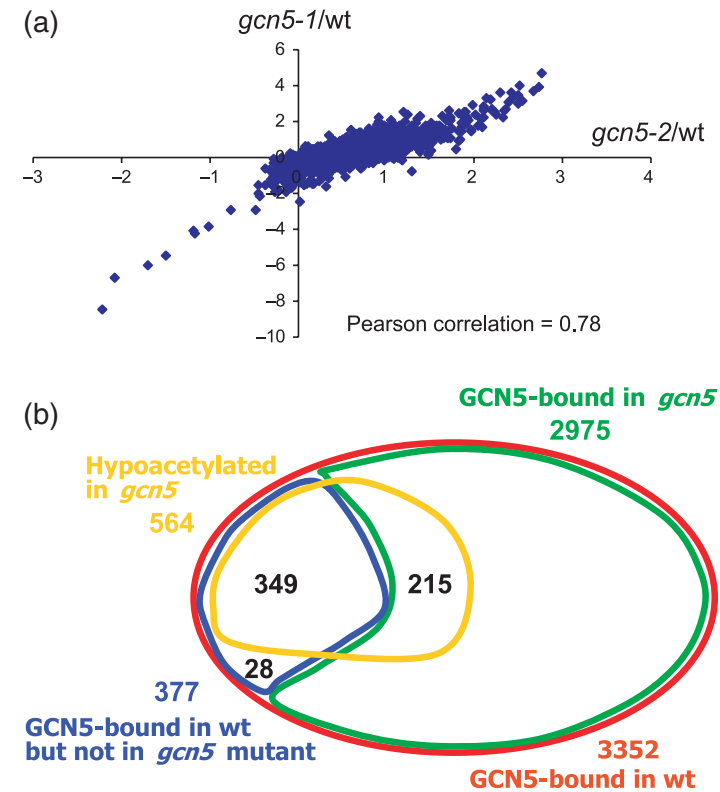

(c)

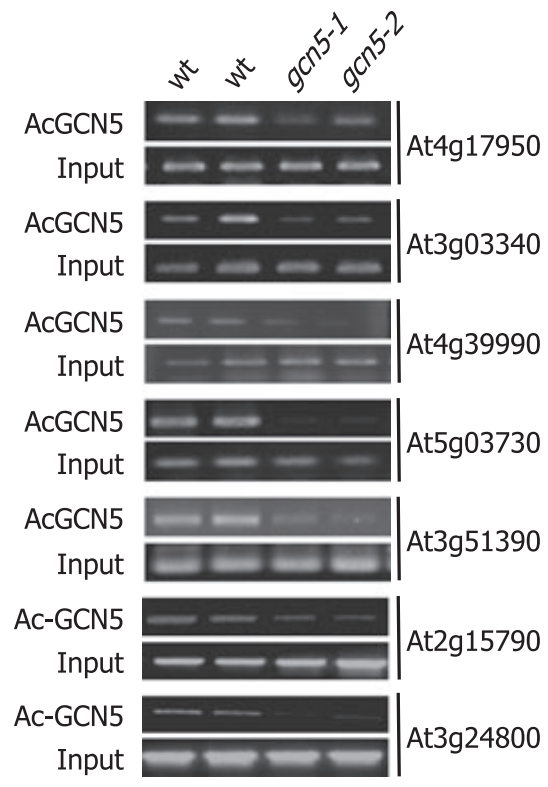

Figure 3. Comparison of promoter targets for GCN5 binding and histone H3K14 acetylation in wild-type, gcn5-1 and gcn5-2 plants.

(a) Comparison between ChIP-chip experiments for wild-type (Ws) versus gcn5-1 or gcn5-2.

(b) Venn diagram representing the intersections between the GCN5 promoter targets in the wild-type and gcn 5 mutants and the promoters showing H3K 14 hypo-acetylation in gcn 5 mutants. All promoters bound by GCN5 in the wildtype are included in the diagram (3352, shown in red). In the gcn5 mutants, most promoter targets remained associated with the GCN5 mutant protein (2975, shown in green), while a smaller proportion were no longer bound (377, shown in blue). All promoters hypo-acetylated in gcn5 mutants versus the wild-type (564, shown in yellow) were bound by GCN5 in the wild-type. Among the promoters that were hypo-acetylated in gcn5 mutants, 215 remained associated with the GCN5 mutant protein but 349 were no longer bound in the gcn5 mutants.

(c) PCR confirmation of ChIP products with primers corresponding to seven promoters enriched in the wild-type compared to gcn5 as determined by ChIP-chip assays.
GCN5 only in the wild-type but not in the gcn5 mutants, or H3K14 hypo-acetylated in the gcn5 mutants, preferentially belonged to gene classes coding for proteins involved in transcription (Figure 4 and Table 1).

\section{GCN5 binding versus transcriptional regulation}

To determine whether GCN5 binding affected transcriptional regulation, we characterized the transcriptome of wild-type, gcn5-1 and gcn5-2 plants by hybridizing CATMA arrays, including 24576 gene-specific tags (Hilson et al., 2004). Wild-type and mutant plants were grown and harvested under the same conditions as those for the ChIP-chip assays. Using two biological replicates, we found that the two mutants had similar transcript profiles (Pearson correlation $=0.77$; calculated for the signal ratios of gcn5-1/wildtype versus gcn5-2/wild-type; Figure S2). In total, 3.5\% (856) of the genes represented by CATMA array features were differentially expressed (Bonferroni $P$-value $<0.05$ ) in both mutants when compared to the wild-type, a percentage close to the $4.0 \%$ for gcn5-1-de-regulated genes identified previously using the Affymetrix Arabidopsis AG array representing 8200 genes (Vlachonasios et al., 2003). Of the gcn5-de-regulated genes, 411 were also present in the SAPv1 array, of which 155 (38\%) are GCN5 binding targets. Among these 155 genes, 97 (63\%) and 58 (37\%) were down and upregulated in the gcn 5 mutants, respectively.

\section{Comparison of GCN5 binding sites with light-regulated gene profiles}

gcn5 plants have a light-hyposensitive phenotype that was most pronounced when grown under continuous far-red light, and certain light-inducible genes have reduced expression and histone acetylation in gcn5 mutants (Benhamed et al., 2006). However, the proportion of promoters corresponding to genes differentially expressed in light or darkness (Ma et al., 2005) was 38\% (467 of 1241 promoters present in the SAPv1 array), almost identical to the genome-wide GCN5 association (Figure 5a).

Recent studies have revealed that the HY5 transcriptional activator of light signaling binds to 3894 genes (Lee et al., 2007). Of these genes, 1475 promoters were present on the SAPv1 array, and 559 (38\%) were bound by GCN5 (38\% of the GCN5 targets). Of the 559 target promoters, 123 are known to be light-regulated (Figure 5a), suggesting that a significant proportion of light-regulated genes depend on binding of both GCN5 and HY5 for expression.

Finally, we compared the GCN5 target promoters with light-regulated gene expression profiles induced by far-red (FR) or red (R) irradiation in a phyA- or phyB-dependent manner (Tepperman et al., 2001, 2004). Genes differentially expressed after 1 and $24 \mathrm{~h}$ are referred to as early and late genes, respectively. When combining both time points, the 


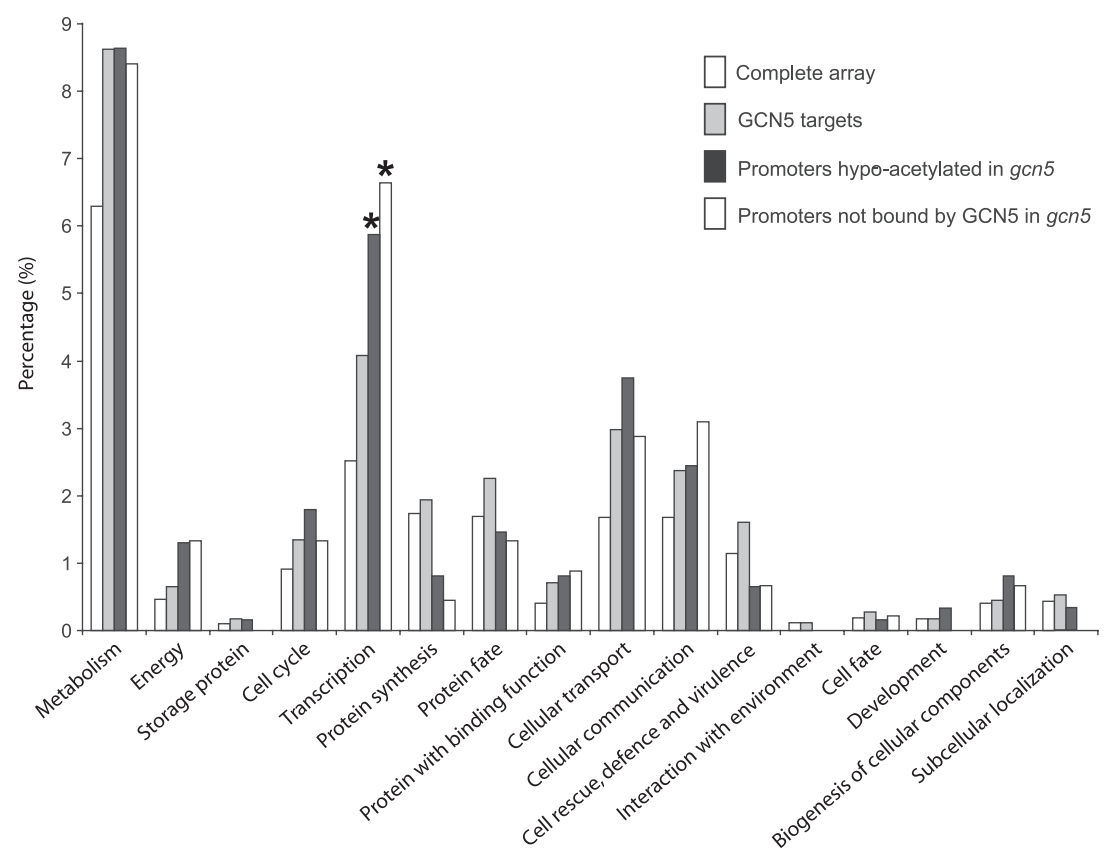

Figure 4. Functional classification of the GCN5 binding target promoters.

Classification was according to the Munich Information Center for Protein Sequences Functiona Categories (FunCat). Asterisks indicate significant bias $\left(\chi^{2}\right.$ test; $\left.P<0.05\right)$ in comparison with the subset represented in the SAPv1 array.

proportions of GCN5 target promoters for genes induced or repressed by $\mathrm{R} / \mathrm{FR}$ irradiation were similar to the genomewide GCN5 association (Figure $5 b$ and data not shown). However, the proportion of GCN5 target promoters was higher in early induced genes and lower in late induced genes, for both $F R$ and $R$ irradiation (Figure $5 b$ and Table S3). No difference was detected between early and late repressed genes (data not shown), similar to the trends observed for HY5 binding targets (Lee et al., 2007).

\section{Discussion}

\section{Multi-purpose Arabidopsis promoter collection}

The SAP repertoire designed in silico contains 22256 Arabidopsis promoter sequences that are fully documented in the SAP online database (http://www.psb. ugent.be/SAP) and the FLAGdb++ genome viewer (http:// urgv.evry.inra.fr/FLAGdb). Of these, 19741 were synthesized by PCR from genomic DNA templates. This sequence repertoire is useful to study diverse aspects of transcriptional regulation and genetic networks. Because each SAP promoter PCR amplicon is flanked by attB4 and attB1 sequences, it can be captured in a plasmid via a Gateway site-specific BP clonase reaction, resulting in a promoter entry clone (Cheo et al., 2004; Dupuy et al., 2004; Sasaki et al., 2004). From such a clone, the promoter sequence can be transferred via an LR clonase reaction into a wide variety of MultiSite Gateway destination vectors, designed for functional assays in plant cells (Karimi et al., 2005, 2007) or in heterologous systems. As a result of the availability of Gateway promoter repertoires, large-scale functional screens have been initiated to dissect the net- works of transcriptional regulators and their target DNA sequences (Deplancke et al., 2004, 2006) or to systematically record in vivo transcriptional activity (Dupuy et al., 2007). Already, over 200 Arabidopsis SAP accessions have been cloned and sequence-verified for downstream applications (unpublished results). In an independent study focusing on genes specifically induced or repressed upon lateral root initiation, transcriptional fusions between each of the 14 tested SAP promoters and the GUS reporter gene confirmed, in transgenic Arabidopsis plants, the pattern deduced from transcript profiles of pericycle sorted cells (Birnbaum et al., 2003; I. De Smet, M. Naudts, R.D., P.H. and T. Beeckman, unpublished results, Department of Plant Systems Biology, Gent, Belgium). This result indicates that, in most cases, promoter sequences of up to $2 \mathrm{~kb}$ are sufficient to recapitulate genuine transcriptional patterns. Additionally, the collection of promoter DNA fragments can be spotted onto microarrays for analysis of chromatin modification or localization of factors associated with chromatin in vivo.

\section{Genome-wide promoter targets of Arabidopsis GCN5}

The promoter targets of the Arabidopsis HAT GCN5 were analyzed by ChIP-chip using the SAPv1 array containing 8471 transcriptionally active promoters. Our data showed that GCN5 targets a large proportion of the promoters in the Arabidopsis genome, distributed homogenously across all five chromosomes, suggesting that GCN5-containing complexes play an essential role in plant transcriptional initiation.

The GCN5 C-terminal bromodomain mediates chromatin association through binding of acetylated histone tails 
Table 1 Promoters of transcription factors bound by GCN5 in the wild-type, but not bound and H3K14 hypo-acetylated in the gcn5 mutants

\begin{tabular}{ll}
\hline AGI code & Gene description \\
\hline At1g06070 & bZIP transcription factor, putative (bZIP69) \\
At1g06850 & Basic leucine zipper 52 \\
At1g12980 & AP2/ERF protein (ESR1) \\
At1g14510 & PHD finger family protein \\
At1g52150 & Member of the class III HD-ZIP protein family INCURV- \\
& ATA 4 \\
At2g03710 & MADS box protein AGL3 SEPALLATA4 \\
At2g21320 & Zinc finger (B-box type) family protein \\
At2g23320 & WRKY DNA-binding protein 15 \\
At2g26990 & Constitutive photomorphogenic 12 \\
At2g30470 & HSI2, high-level expression of sugar-inducible 2 \\
At2g35310 & Transcriptional factor B3 family protein \\
At3g12720 & Member of the R2R3 factor gene family \\
At3g27260 & DNA-binding bromodomain-containing protein GTE8 \\
At3g28210 & Putative zinc finger protein (PMZ) \\
At3g46130 & Putative transcription factor MYB48 \\
At3g53500 & RSZ32 nucleic acid binding \\
At3g54220 & Scarecrow transcription factor, putative SGR1 \\
At3g54620 & Basic leucine zipper 25 \\
At3g55370 & Zinc finger protein OBP3 \\
At3g57670 & Zinc finger (C2H2 type) family protein \\
At3g61950 & Basic helix-loop-helix (bHLH) family protein \\
At3g61970 & DNA-binding protein NGA2 (NGATHA2) \\
At3g62340 & WRKY transcription factor WRKY68 \\
At4g07950 & DNA-directed RNA polymerase III family protein \\
At4g14220 & Zinc finger (C3HC4-type RING finger) family protein \\
At4g17950 & DNA-binding family protein \\
At4g29000 & Tesmin/TSO1-like CXC domain-containing protein \\
At4g32800 & Member of the DREB subfamily A-4 \\
At4g32850 & Poly(A) polymerase IV \\
At5g02030 & BEL1-like homeodomain 8 \\
At5g44080 & bZIP transcription factor family protein \\
At5g46350 & WRKY transcription factor \\
At5g51190 & ERF (ethylene response factor) subfamily B-3 \\
At5g59450 & Scarecrow-like transcription factor 11 (SCL11) \\
At5g59780 & Putative transcription factor MYB59 \\
At5g63280 & Zinc finger (C2H2 type) family protein \\
At5g67110 & Myc/bHLH transcription factor-like protein ALCATRAZ \\
\hline &
\end{tabular}

(Imoberdorf et al., 2006; Mujtaba et al., 2007), but its disruption in two T-DNA insertion mutants abolished binding to only $11 \%$ of the GCN5 promoter targets, implying that the bromodomain is not necessary for recruitment to most target promoters in Arabidopsis. Therefore, association with GCN5 probably also relies on additional interactions with DNA-binding transcription factors or with other subunits in various HAT complexes, as direct GCN5-DNA interaction has not been reported to date.

\section{Binding of GCN5 to target promoters does not always correlate with transcriptional regulation}

Our data revealed that partial or full deletion of the GCN5 bromodomain resulted in de-regulation of $3.5 \%$ of the Arabidopsis genes. Only $38 \%$ (155/411) of the de-regulated (a) HY5-bound Light regulated

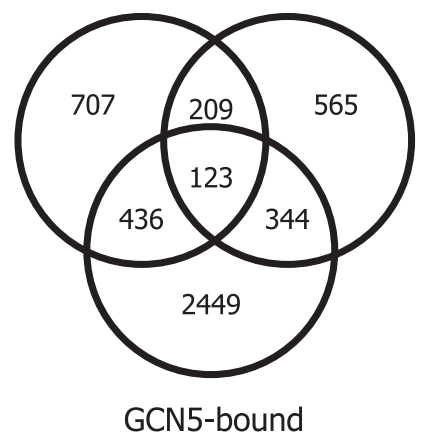

(b)
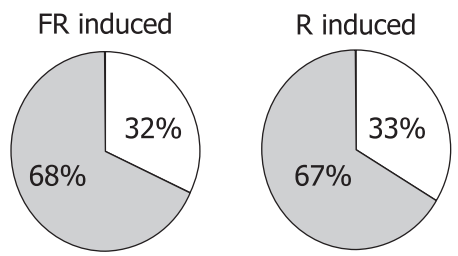

FR early induced
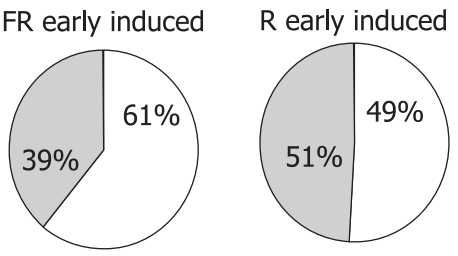

FR late induced

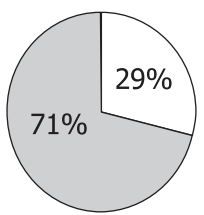

$\mathrm{R}$ late induced

GCN5-bound

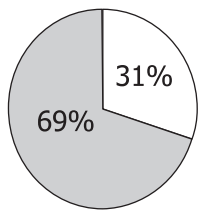

Not bound by GCN5

Figure 5. GCN5 binding to the promoters of light-regulated genes and its relationship with the light-signaling transcriptional activator HY5. (a) Venn diagram comparing light-regulated genes with the binding targets of GCN5 and HY5. Of the light-regulated genes (Ma et al., 2005), 1241 were represented on the SAPv1 array, of which 467 were GCN5 targets. Of the HY5 targets (Lee et al., 2007), 1475 were represented on the SAPv1 array, of which 559 were GCN5 targets, and 123 light-regulated genes were targets of both GCN5 and HY5.

(b) Comparison between fraction of promoters bound by GCN5 for early and late genes induced by FR or $\mathrm{R}$ light at an early or late stage. The differences between early- and late-induced GCN5 targets were statistically significant ( $\chi^{2}$ test).

genes overlapped with the GCN5 targets, suggesting that de-regulation of most genes is an indirect consequence of the gcn5 mutations. For example, the gcn5 mutations might affect the global histone acetylation level that is required for expression of gene subsets, because the bromodomain might be involved in propagation of chromatin acetylation (Imoberdorf et al., 2006; Mujtaba et al., 2007).

In contrast, gcn5 mutations did not alter the expression of $62 \%$ of the GCN5 target genes. Indeed, GCN5 binding 
does not always affect gene transcription, as previously illustrated by its constitutive association with light-responsive promoters irrespective of light conditions (Benhamed et al., 2006). In such cases, specific signaling pathways might regulate GCN5 activity in response to cellular cues. Alternatively, GNC5 may be associated with nucleosomes positioned in chromatin regions that do not regulate the transcriptional activity of neighboring genes, and may be located within or outside the genome fragments included in the SAP promoter amplicons. That gcn5-1 and gcn5-2 are not null alleles might also be the reason for the lack of $g c n 5$ transcriptional de-regulation: both mutants still produce truncated polypeptides (Figure S1), and only the gcn5-2 mutation removes the entire bromodomain.

Nevertheless, $37 \%$ (58/155) of the gcn5-de-regulated genes are induced in the mutants (Figure 5), possibly due to indirect effects mediated by the de-regulated transcription factors. Alternatively, GCN5 might exert a transcriptional repressor activity on some genes that could be mediated by acetylation of protein substrates other than histones, such as DNA-binding factors or general factors of the transcription machinery. Finally, HAT activities could also be required for nucleosome assembly during repression, as it is for the developmental regulation of yeast genes (Recht et al., 2006).

\section{Bromodomain involvement in promoter binding and H3K14 acetylation}

Bromodomains are evolutionarily conserved proline-rich protein modules that are found mostly in HAT complexes and that interact with the $\mathrm{N}$-terminal tails of histones $\mathrm{H} 3$ and $\mathrm{H} 4$ with acetylated lysines. The gcn5 mutations affect acetylation of several histone $\mathrm{H} 3$ lysine residues, including $\mathrm{K} 9$, K14 and K27, on light-inducible gene promoters (Benhamed et al., 2006). Acetylation of H3K9 and H3K27, however, was also reduced by mutation of TAF1, which is another HAT gene that is involved in light-controlled transcriptional regulation (Bertrand et al., 2005). Therefore, H3K14 acetylation of the tested light-responsive promoters appears to depend specifically on the intact bromodomain of GCN5. Only $7 \%(546 / 8471)$ of the SAPv1 promoters were H3K14 hypo-acetylated in the gcn5 mutants, indicating that, for most promoters in the genome, the GCN5 bromodomain is not essential for this modification. Alternatively, the bromodomain might be required for $\mathrm{H} 3 \mathrm{~K} 14$ acetylation of the ORF during transcriptional elongation, which is mediated by the GCN5-containing SAGA HAT complex (Govind et al., 2007). We cannot exclude the possibility that the GCN5 bromodomain is also involved in global maintenance of chromatin acetylation but not in modification of specific loci. Interestingly, 93\% (349/377) of the target promoters that required the bromodomain for GCN5 binding also showed H3K14 hypo-acetylation in the gcn5 mutants. Thus, for a subset of GCN5 promoter targets, GCN5 recruitment and H3K14 acetylation are coupled and controlled by the GCN5 bromodomain.

\section{GCN5 in light regulation}

GCN5 is required for light-regulated gene expression and hypocotyl elongation (Benhamed et al., 2006). We found that the ratio of GCN5-bound versus non-bound targets was similar across all promoters tested $(40 \%)$ and among lightregulated genes (38\%). However, $61 \%$ and $49 \%$ of the early gene promoters induced by FR and R light, respectively, are GCN5 binding targets. HY5, which has been proposed to function as a high hierarchical regulator of the transcriptional cascade controlling photomorphogenesis, also targets more than $60 \%$ of the early FR- and R-induced genes (Lee et al., 2007). Recent studies have shown that acetylation of histones in the yeast PHO5 gene by a HAT complex under repressed conditions (high phosphate) is essential for the binding of $\mathrm{PHO} 4$, a DNA-binding transcription factor that is activated upon phosphate starvation (Nourani et al., 2004). Similarly, GCN5 might prime for later activation of lightinducible genes in the dark repressed state. Because a large proportion of $\mathrm{HY} 5$ target promoters induced early by FR or $\mathrm{R}$ light were also targets of GCN5 (Table S3), HY5 and GCN5 might cooperate to induce expression of these genes. In support of this hypothesis, genetic interactions between HY5 and GCN5 have been found in the regulation of photomorphogenesis (Benhamed et al., 2006). Whether GCN5 and HY5 interact directly remains to be established.

\section{Experimental procedures}

\section{In silico design of promoter amplicons}

Promoter sequences were extracted from the intergenic regions defined in the genome annotation of the Arabidopsis Information Resource (TAIR version 5). Only sequences upstream of translation initiation codons were considered, thereby discarding intergenic regions between two convergent genes. Genes described as putative transposable elements or parts thereof were also eliminated. When divergent, both promoters derived from the same intergenic region were selected independently and relative to their respective translational start site. For annotated splice variants, only the largest gene model was considered.

In all cases, the $3^{\prime}$ primer defining the promoter amplicon was positioned immediately next to, but excluding, the translation initiation codon, and only its length could vary when searching for a matching PCR primer pair. In most cases, the $5^{\prime}$ primer was positioned close to the next upstream coding sequence when the intergenic region was $<2 \mathrm{~kb}$, or approximately $2 \mathrm{~kb}$ upstream of the cognate translation initiation codon when the intergenic region was $>2 \mathrm{~kb}$. In a minority of cases $(1128 ; 5.5 \%)$, the designed promoter amplicons were $>2.2 \mathrm{~kb}$ and included the entire intergenic region. The $5^{\prime}$ primer was selected within a window centered on the desired boundary and approximately $10 \%$ of the target size, thereby enabling some flexibility in the search for matching PCR primers. PCR primers were designed in three successive rounds 
using software based on the OSP.pm perl library: (i) primers with size ranging from 18 to 28 nucleotides; (ii) primers with maximum size increased to 32 nucleotides; (iii) primers with melting temperature range relaxed from $55-65$ to $50-70^{\circ} \mathrm{C}$. The resulting primer pairs were tested for specificity across the whole genome. Each promoter-specific primer sequence (average 28 nucleotides) was extended at the $5^{\prime}$ end by 15 bases, including a trinucleotide specificity tag (TST) and a portion of the attB4 and attB1 Gateway recombination site in the $5^{\prime}$ and $3^{\prime}$ primer, respectively (Figure 1 and Table S4).

\section{Promoter synthesis}

SAPv1 and SAPv2 were initially amplified in a two-step PCR (Figure 1). In the first step, $50 \mathrm{ng}$ of Col- 0 genomic DNA and $1 \mu \mathrm{m}$ of paired specific primers were resuspended in a $10 \mu \mathrm{l}$ final volume of PCR mix with 1 unit of Platinum Taq high-fidelity DNA polymerase (Invitrogen, http://www.invitrogen.com), and incubated for $2 \mathrm{~min}$ at $94^{\circ} \mathrm{C}$, then for $11 \mathrm{cycles}$ of $30 \mathrm{sec}$ at $94^{\circ} \mathrm{C}, 30 \mathrm{sec}$ at $55^{\circ} \mathrm{C}$ and $2 \mathrm{~min}$ at $68^{\circ} \mathrm{C}$, then held at $4^{\circ} \mathrm{C}$. In the second step, $40 \mu \mathrm{l}$ of PCR mix was added to the initial reaction, containing $5 \mu \mathrm{m}$ of the universal attB4TST and attB1TST oligonucleotides and 1 unit of fresh platinum Taq high-fidelity DNA polymerase, and incubated $2 \mathrm{~min}$ at $94^{\circ} \mathrm{C}$, then for 30 cycles of $30 \mathrm{sec}$ at $94^{\circ} \mathrm{C}$, $30 \mathrm{sec}$ at $55^{\circ} \mathrm{C}$ and $2 \mathrm{~min}$ at $68^{\circ} \mathrm{C}$, followed by $5 \mathrm{~min}$ at $68^{\circ} \mathrm{C}$, then held at $4^{\circ} \mathrm{C}$. The 40 universal primers carried either the full attB4 or attB1 Gateway recombination site and the $3^{\prime}$ TST defining each of the 16 rows or 24 columns of a 384-well plate (Table S4). These 40 primers were sufficient for re-amplification of the entire SAP collection. TSTs distinguished rows and columns and helped resolve potential well-to-well cross-contamination. The SAP promoter amplicon collection is available upon request.

Subsequent SAPv1 PCR amplifications were carried out using $2 \mu \mathrm{l}$ of a threefold dilution in double-distilled water $\left(\mathrm{ddH}_{2} \mathrm{O}\right)$ of the primary product as template, 2 units of Taq DNA polymerase in a $100 \mu \mathrm{l}$ final volume, and the following program: $2 \mathrm{~min}$ at $94^{\circ} \mathrm{C}, 30$ cycles of $30 \mathrm{sec}$ at $94^{\circ} \mathrm{C}, 30 \mathrm{sec}$ at $55^{\circ} \mathrm{C}$ and $2 \mathrm{~min}$ at $68^{\circ} \mathrm{C}$, then $15 \mathrm{~min}$ at $68^{\circ} \mathrm{C}$, then held at $4^{\circ} \mathrm{C}$. To assess the success of the PCR, $8 \mu \mathrm{l}$ from each reaction was loaded on a $2 \%$ agarose gel, and the size of every amplicon was verified. SAP amplicons were purified on Multiscreen plates (Millipore, http://www.millipore.com) and resuspended in Tris/EDTA/dimethyl sulfoxide at approximately $100 \mathrm{ng} \mathrm{ml}^{-1}$. The purified probes were transferred to 1536-well plates using a Genesis workstation (TECAN, http://www.tecan.com). Although amplification did not yield a detectable product of the expected size in all cases, promoter probes were not rearranged to avoid arraying errors. Each spot was flagged with a PCR product quality code: 0 , not detected $(3317,27.9 \%) ; 1$, expected size (8471, $71.2 \%) ; 2$, wrong size $(116,1 \%)$.

\section{SAP array production}

Amplicons were spotted on UltraGAPS slides (Corning, http:// www.corning.com) by means of a Microgrid II (Genomic Solutions, http://www.genomicsolutions.com) using a 64-pin tool. The array design is fully documented as array accession number A-MEXP-817 in the ArrayExpress repository (http://www.ebi.ac.uk/ arrayexpress/). Pin performance and print quality (spot diameter and homogeneity) were assessed before each SAP DNA printing run. For each batch, quality was controlled on two SAPv1 arrays colored with POPO-3 iodide (Invitrogen). Only arrays with $>99 \%$ good spots were further processed (same quality threshold as for CATMA arrays).

\section{Plant material}

The gcn5-1 (Vlachonasios et al., 2003) and gcn5-2 (Bertrand et al., 2003) T-DNA insertion mutant alleles of Arabidopsis thaliana (L.) Heyhn. are both in the Wassileskija (Ws) ecotype. Prior to germination, seeds of Ws, gcn5- 1 and gcn5-2 were sterilized and stratified for 2 days at $4^{\circ} \mathrm{C}$. For in vitro cultures, seeds were sown on $0.5 \mathrm{X}$ Murashige and Skoog medium, incubated at $4^{\circ} \mathrm{C}$ for $48 \mathrm{~h}$, and transferred to growth chambers for germination at $20^{\circ} \mathrm{C}$ under white light ( $16 \mathrm{~h}$ at $120 \mu \mathrm{mol} \mathrm{m} \mathrm{m}^{-2} \mathrm{sec}^{-1}$ ).

\section{Protein gel blots}

For protein gel blots, nuclear proteins were extracted from wild-type or gcn 5 plants by grinding rosette leaves to a fine powder in liquid nitrogen. The powder was transferred to nuclear extraction buffer (0.4 M sucrose, $10 \mathrm{~mm}$ Tris $/ \mathrm{HCl} \mathrm{pH} \mathrm{8,} 10 \mathrm{~mm} \mathrm{MgCl}, 5 \mathrm{~mm} \beta$-mercaptoethanol and $0.1 \mathrm{~mm}$ PMSF). The extracts were filtered twice through Miracloth (Calbiochem, http://www.emdbiosciences.com), and centrifuged at $4000 \mathrm{~g}$ for $20 \mathrm{~min}$ at $4^{\circ} \mathrm{C}$. The nuclear pellets were dissolved in $15 \%$ SDS-PAGE gel-loading buffer for electrophoretic analysis. The blots were incubated with the GCN5 antibody (1:500 dilution) overnight at $4^{\circ} \mathrm{C}$, and revealed by enhanced chemiluminescence (GE Healthcare, http://www.gehealthcare.com).

\section{Chromatin immunopreciptation and SAP array hybridization}

Immunoprecipitations were performed as described previously (Gendrel et al., 2005). Twelve-day-old seedlings grown under longday conditions were harvested at developmental stage 1.03 (Boyes et al., 2001), $4 \mathrm{~h}$ after dawn, fixed in $1 \%$ formaldehyde for $15 \mathrm{~min}$ in a vacuum, and neutralized with $0.125 \mathrm{M}$ glycine. After washing with sterilized water, samples were ground in liquid nitrogen as described above. Nuclear pellets were suspended in a buffer containing $0.25 \mathrm{~m}$ sucrose, $10 \mathrm{~mm}$ Tris $/ \mathrm{HCl} \mathrm{pH} 8,10 \mathrm{~mm} \mathrm{MgCl}_{2}, 1 \%$ Triton X-100, $5 \mathrm{~mm} \beta$-mercaptoethanol, $0.1 \mathrm{~mm}$ PMSF and protease inhibitors (one mini-tablet per ml; Roche Diagnostics, http:// www.roche.com). The suspensions were transferred to microfuge tubes and centrifuged at $12000 \mathrm{~g}$ for $10 \mathrm{~min}$. The pellets were suspended in $1.7 \mathrm{~m}$ sucrose, $10 \mathrm{~mm}$ Tris/ $\mathrm{HCl} \mathrm{pH} 8,2 \mathrm{~mm} \mathrm{MgCl}_{2}$, $0.15 \%$ Triton X-100, $5 \mathrm{~mm} \beta$-mercaptoethanol, $0.1 \mathrm{~mm}$ PMSF and protease inhibitors, and centrifuged at $12000 \mathrm{~g}$ for $1 \mathrm{~h}$ through a layer of the same buffer in microfuge tubes. The nuclear pellets were lysed in a buffer containing $50 \mathrm{~mm}$ Tris/ $\mathrm{HCl} \mathrm{pH} \mathrm{8,} 10 \mathrm{~mm}$ EDTA, $1 \%$ SDS and protease inhibitors. The lysed nuclei were sonicated four times for $15 \mathrm{sec}$ at $4^{\circ} \mathrm{C}$, followed by centrifugation at $12000 \mathrm{~g}$ for $10 \mathrm{~min}$. The supernatants containing chromatin fragments were diluted 10 -fold with 1\% Triton X-100, 1.2 mm EDTA, 16.7 mm Tris/ $\mathrm{HCl} \mathrm{pH} \mathrm{8,} \mathrm{and} 167 \mathrm{~mm} \mathrm{NaCl}$. A $1 \mathrm{ml}$ aliquot of the dilution was used per immunoprecipitation assay. Antibodies against acetyl histone H3K14 and GCN5 were purchased from Upstate Biotechnology (http://www.millipore.com) and custom-prepared in rabbits (Eurogentec, http://www.eurogentec.com; Benhamed et al., 2006), respectively.

Labeling and hybridization were adapted from the methods described by Gendrel et al. (2005). DNA from the input chromatin fraction prior to immunoprecipitation (input control) or recovered after ChIP (IP fraction) was differentially labeled and hybridized in classical dye swap experiments to correct for dye bias. The IP and input fractions were precipitated and amplified as follows. The first step was carried out in $16.5 \mu \mathrm{l}$ final volume: $2 \mathrm{~min}$ at $94^{\circ} \mathrm{C}, 10 \mathrm{~min}$ at $5^{\circ} \mathrm{C}$, addition of the mix containing primer $\mathrm{A}\left(5^{\prime}\right.$-GTTTCCCAGTCACGATCNNNNNNNNN- $\left.3^{\prime}\right), 8$ min at $37^{\circ} \mathrm{C}, 2$ min at $94^{\circ} \mathrm{C}, 5 \mathrm{~min}$ at $10^{\circ} \mathrm{C}$, 
addition of $4 \mu \mathrm{l}$ Sequenase (USB Corporation, http://www. usbweb.com), $8 \mathrm{~min}$ at $37^{\circ} \mathrm{C}$, then held at $4^{\circ} \mathrm{C}$. After addition of $43.5 \mu \mathrm{lddH_{2 }}$ O, $15 \mu \mathrm{l}$ of the first reaction dilution were used as template for the amplification step, after addition of primer $B\left(5^{\prime}\right.$ GTTTCCCAGTCACGATC- $3^{\prime}$ ), in $100 \mu \mathrm{l}$ final volume, with the following program: $3 \mathrm{~min}$ hold at $94^{\circ} \mathrm{C} ; 30$ cycles of $30 \mathrm{sec}$ at $94^{\circ} \mathrm{C}, 30 \mathrm{sec}$ at $40^{\circ} \mathrm{C}, 30 \mathrm{sec}$ at $50^{\circ} \mathrm{C}$ and $1 \mathrm{~min}$ at $72^{\circ} \mathrm{C}$; then held at $4^{\circ} \mathrm{C}$.

PCR products were purified using Qiaquick columns (Qiagen, http://www.qiagen.com) according to the manufacturer's instructions, and labeled with either Cy3-dCTP or Cy5- dCTP (NEN, las.perkinelmer.com) using 10 units of DNA Klenow polymerase and random hexamers. Labeled samples were purified using Qiaquick columns, quantified, combined and precipitated. At least $40 \mathrm{pmol}$ of each labeled sample was used per hybridization. Slides were pre-hybridized for $1 \mathrm{~h}$, hybridized overnight at $42^{\circ} \mathrm{C}$ in $25 \%$ formamide, washed in $2 \times \mathrm{SSC}, 0.1 \%$ SDS for 4 min at $42^{\circ} \mathrm{C}$, and in $1 \times$ SSC for $4 \mathrm{~min}, 0.2 \times$ SSC for $4 \mathrm{~min}$ and $0.05 \times$ SSC for $4 \mathrm{~min}$ at room temperature, and dried by centrifugation at $1000 \mathrm{~g}$ for $2 \mathrm{~min}$. A technical replicate in the dye swap was used for each comparison. The arrays were scanned using a GenePix 4000A scanner (Axon Instruments, http://www.moleculardevices.com), images were analyzed with the software GenePix Pro 3.0 (Axon Instruments), and data were stored as a GenePix reader (gpr) file. The ChIP-chip data have been submitted to Array Express under accession number E-MEXP-1233.

\section{ChIP-chip SAPv1 array data analysis}

Pre-processing data and normalization were as described previously (Turck et al., 2007). Raw data ( $\log _{2}$ of the median feature pixel intensity as listed in gpr files, without background subtraction) for the 635 and $532 \mathrm{~nm}$ signals were processed, excluding spots manually flagged because of sub-standard features. Array-by-array normalization, such as loess, could not be applied because the signal distribution of the input and IP samples differed substantially. Instead, data were normalized across arrays based on the properties of the dye swap. As the second array was a technical replicate of the first one, the spot signal of the sample hybridized on the first array and labeled with the red dye (or the green dye) should be close to the green signal of the second array (or the red signal, respectively). In practice, the relationship is linear, but it is not the identity function. To correct this gap, we used these following linear models: $Y_{21 s}=a+b Y_{12 s}+N\left(0, \sigma^{2}\right)$ and $Y_{22 s}=c+d Y_{11 s}+N\left(0, \sigma^{2}\right)$, where $Y_{i j s}$ is the signal of the spot ' $s$ ' when the sample hybridized to array ' $i$ ' is labeled with dye ' $j$ ', and the normalized IP and input values of spot 's' on the second array relative to the first one are defined by $Y_{21 s}=\left(Y_{21 s}-a\right) / b$ and $Y_{22 s}=\left(Y_{22 s}-c\right) / d$, respectively. Then the values of the two arrays in the same dye-swap were averaged for each sample and for each spot.

For each dye swap, 'enriched' probes were defined as probes yielding a signal significantly higher than the background. To this end, the IP signal histogram was modeled according to mixture models. Truncated and non-truncated Gaussian mixture models were applied, with a component number varying from 1 to 5 . Models with two or three components were systematically selected using the Bayesian information criterion. The components were interpreted as determining significant IP signals. The selected model was often a mixture with two well separated components. In such a case, probes classified in the component with the greatest mean according to the maximum a posteriori (MAP) rule were considered to yield a significant IP value. When the number of components was three, the component with the lowest mean was well separated from the others and characterized background hybridization. Therefore, probes classified in the other two compo- nents according to the MAP rule were considered to yield a significant IP value. A promoter is considered bound when it yields significant IP values in the three biological replicates.

\section{Transcriptome CATMA array hybridization}

Transcriptome profiling was analyzed using Arabidopsis CATMA arrays containing 24576 gene-specific tags (CATMAv2 repertoire; Crowe et al., 2003; Hilson et al., 2004). Arrays were printed and hybridized as described previously (Lurin et al., 2004). RNA was extracted from 12-day-old seedlings at developmental stage 1.03 grown under long-day conditions (Boyes et al., 2001). Each experiment was carried out with two independent biological repeats to take into account biological variation. Each comparison included a technical repeat with fluorochrome reversal (dye swap) for each pool of RNA. RNA integrity was verified using a Bioanalyzer (Agilent, http://www.agilent.com). For each sample, cRNAs were synthesized from $2 \mu \mathrm{g}$ of total RNA using the Message Amp aRNA kit (Ambion, http://www.ambion.com), and $5 \mu \mathrm{g}$ of cRNA was reversetranscribed using 300 units of SuperScript II (Invitrogen) and Cy3-dUTP or Cy5-dUTP (NEN) for each slide. Labeled samples were combined, purified, and concentrated using YM30 Microcon columns (Millipore). The CATMA arrays were pre-hybridized, hybridized, washed, dried and scanned as for SAPv1 arrays. The transcript profiling data have been submitted to Array Express under accession number E-MEXP-1241.

\section{CATMA array data analysis}

Transcriptome statistical analysis was based on dye swaps (i.e. two arrays each containing 24576 GSTs and 384 controls). For each array, the raw data consisted of the $\log _{2}$ median feature pixel intensity without background subtraction, at $635 \mathrm{~nm}$ (red) and $532 \mathrm{~nm}$ (green). Data analysis was performed as described previously (Gagnot et al., 2008). In the following description, log ratio refers to the differential expression between two conditions: either $\log _{2}$ red/green or $\log _{2}$ green/red according to the experimental design. Array-by-array normalization was performed to remove systematic biases: spots with poor features were excluded; to correct for dye bias, signal was normalized globally according to intensity using the LOESS procedure; and, for each block, the log ratio median calculated over the values for the entire block was subtracted from each individual log ratio value to correct for print tip effects in each metablock. To determine differentially expressed genes, the log ratios were analyzed using a paired $t$-test, assuming that the variance of the log ratios is the same for all genes. Spots with extreme variance (high or low) were excluded. The raw $P$ values were adjusted by the Bonferroni method (type I error equal to $5 \%$ ) to control for family-wise error rate and to limit false-positives drastically in a multiple comparison context (Benjamini and Hochberg, 1995). The data were deposited in the ArrayExpress (http:// www.ebi.ac.uk/arrayexpress/) according to MIAME standards.

\section{Acknowledgements}

The SAP consortium efforts are supported by Génoplante (phase III, program M04) for array printing, hybridization and analysis through a grant to J.P.R., and by grants from the Stiftelsen for Strategisk Forskning to R.P.B., from the Division of Earth and Life Sciences of the Netherlands Research Council (The Hague, the Netherlands), from the ETH Zurich and the Swiss National Science Foundation (3100A0-109475) to W.G., and by the Max Planck Society (through 
Detlef Weigel, Max Planck Institute for Developmental Biology, Tübingen, Germany) for generation of the promoter resource. We thank Martine De Cock for help in preparing the manuscript and Karel Spruyt for assistance with illustrations.

\section{Supporting Information}

Additional Supporting Information may be found in the online version of this article:

Figure S1. Protein gel analysis of GCN5 proteins from whole-cell extracts of the wild-type (Ws) and gcn5 mutants.

Figure S2. Comparison of transcript profiles between gcn5-1, gcn5-2 and the wild-type.

Table S1. Transcripts and chromatin targets that differ between the wild-type and gcn5 mutants.

Table S2. Primers for PCR analysis of GCN5 chromatin immunoprecipitation.

Table S3. GCN5 and HY5 binding with respect to light transcriptional regulation.

Table S4. Universal primers for SAP promoter secondary amplification.

Please note: Wiley-Blackwell are not responsible for the content or functionality of any supporting materials supplied by the authors. Any queries (other than missing material) should be directed to the corresponding author for the article.

\section{References}

Benhamed, M., Bertrand, C., Servet, C. and Zhou, D.-X. (2006) Arabidopsis GCN5, HD1, and TAF1/HAF2 interact to regulate histone acetylation required for light-responsive gene expression. Plant Cell, 18, 2893-2903.

Benjamini, Y. and Hochberg, Y. (1995) Controlling the false discovery rate: a practical and powerful approach to multiple testing. J. $R$ Stat. Soc. B, 57, 289-300.

Bertrand, C., Bergounioux, C., Domenichini, S., Delarue, M. and Zhou, D.-X. (2003) Arabidopsis histone acetyltransferase AtGCN5 regulates the floral meristem activity through the WUSCHEL/ AGAMOUS pathway. J. Biol. Chem. 278, 28246-28251.

Bertrand, C., Benhamed, M., Li, Y.-F., Ayadi, M., Lemonnier, G., Renou, J.-P., Delarue, M. and Zhou, D.-X. (2005) Arabidopsis HAF2 gene encoding TATA-binding protein (TBP)-associated factor TAF1, is required to integrate light signals to regulate gene expression and growth. J. Biol. Chem. 280, 1465-1473.

Birnbaum, K., Shasha, D.E., Wang, J.Y., Jung, J.W., Lambert, G.M., Galbraith, D.W. and Benfey, P.N. (2003) A gene expression map of the Arabidopsis root. Science, 302, 1956-1960.

Boyes, D.C., Zayed, A.M., Ascenzi, R., McCaskill, A.J., Hoffman, N.E., Davis, K.R. and Görlach, J. (2001) Growth stage-based phenotypic analysis of Arabidopsis: a model for high throughput functional genomics in plants. Plant Cell, 13, 1499-1510.

Carré, C., Szymczak, D., Pidoux, J. and Antoniewski, C. (2005) The histone H3 acetylase dGcn5 is a key player in Drosophila melanogaster metamorphosis. Mol. Cell Biol. 25, 8228-8238.

Cheo, D.L., Titus, S.A., Byrd, D.R.N., Hartley, J.L., Temple, G.F. and Brasch, M.A. (2004) Concerted assembly and cloning of multiple DNA segments using in vitro site-specific recombination: functional analysis of multi-segment expression clones. Genome Res. 14, 2111-2120.

Cosma, M.P., Tanaka, T. and Nasmyth, K. (1999) Ordered recruitment of transcription and chromatin remodeling factors to a cell cycle- and developmentally regulated promoter. Cell, 97, 299311.
Crowe, M.L., Serizet, C., Thareau, V. et al. (2003) CATMA: a complete Arabidopsis GST database. Nucleic Acids Res. 31, 156-158.

Deplancke, B., Dupuy, D., Vidal, M. and Walhout, A.J.M. (2004) A Gateway-compatible yeast one-hybrid system. Genome Res. 14, 2093-2101.

Deplancke, B., Mukhopadhyay, A., Ao, W. et al. (2006) A gene-centered C. elegans protein-DNA interaction network. Cell, 125, 1193-1205.

Dupuy, D., Li, Q.-R., Deplancke, B. et al. (2004) A first version of the Caenorhabditis elegans promoterome. Genome Res. 14, 21692175.

Dupuy, D., Bertin, N., Hidalgo, C.A. et al. (2007) Genome-scale analysis of in vivo spatiotemporal promoter activity in Caenorhabditis elegans. Nat. Biotechnol. 25, 663-668.

Gagnot, S., Tamby, J.P., Martin-Magniette, M.L., Bitton, F., Taconnat, L., Balzergue, S., Aubourg, S., Renou, J.P., Lecharny, A. and Brunaud, V. (2008) CATdb: a public access to Arabidopsis transcriptome data from the URGV-CATMA. Nucleic Acids Res. 36, D986-D990.

Gendrel, A.-V., Lippman, Z., Martienssen, R. and Colot, V. (2005) Profiling histone modification patterns in plants using genomic tiling microarrays. Nat. Methods, 2, 213-218.

Govind, C.K., Zhang, F., Qiu, H., Hofmeyer, K. and Hinnebusch, A.G. (2007) Gcn5 promotes acetylation, eviction, and methylation of nucleosomes in transcribed coding regions. Mol. Cell, 25, 31-42.

Hanlon, S.E. and Lieb, J.D. (2004) Progress and challenges in profiling the dynamics of chromatin and transcription factor binding with DNA microarrays. Curr. Opin. Genet. Dev. 14, 697-705.

Hilson, P., Allemeersch, J., Altmann, T. et al. (2004) Versatile genespecific sequence tags for Arabidopsis functional genomics: transcript profiling and reverse genetics applications. Genome Res. 14, 2176-2189.

Horn, P.J. and Peterson, C.L. (2002) Chromatin higher order folding: wrapping up transcription. Science, 297, 1824-1827.

Imoberdorf, R.M., Topalidou, I. and Strubin, M. (2006) A role for Gcn5-mediated global histone acetylation in transcriptional regulation. Mol. Cell. Biol. 26, 1610-1616.

Jenuwein, T. and Allis, C.D. (2001) Translating the histone code. Science, 293, 1074-1080.

Karimi, M., De Meyer, B. and Hilson, P. (2005) Modular cloning in plant cells. Trends Plant Sci. 10, 103-105.

Karimi, M., Bleys, A., Vanderhaeghen, R. and Hilson, P. (2007) Building blocks for plant gene assembly. Plant Physiol. 145, 11831191.

Lee, K.K. and Workman, J.L. (2007) Histone acetyltransferase complexes: one size doesn't fit all. Nat. Rev. Mol. Cell Biol. 8, 284-295.

Lee, T.I., Causton, H.C., Holstege, F.C.P., Shen, W.-C., Hannett, N., Jennings, E.G., Winston, F., Green, M.R. and Young, R.A. (2000) Redundant roles for the TFIID and SAGA complexes in global transcription. Nature, 405, 701-704.

Lee, J., He, K., Stolc, V., Lee, H., Figueroa, P., Gao, Y., Tongprasit, W., Zhao, H., Lee, I. and Deng, X.W. (2007) Analysis of transcription factor HY5 genomic binding sites revealed its hierarchical role in light regulation of development. Plant Cell, 19, 731-749.

Long, J.A., Ohno, C., Smith, Z.R. and Meyerowitz, E.M. (2006) TOPLESS regulates apical embryonic fate in Arabidopsis. Science, 312, 1520-1523.

Lurin, C., Andrés, C., Aubourg, S. et al. (2004) Genome-wide analysis of Arabidopsis pentatricopeptide repeat proteins reveals their essential role in organelle biogenesis. Plant Cell, 16, 20892103. 
Ma, L., Sun, N., Liu, X., Jiao, Y., Zhao, H. and Deng, X.W. (2005) Organ-specific expression of Arabidopsis genome during development. Plant Physiol. 138, 80-91.

Mao, Y., Pavangadkar, K.A., Thomashow, M.F. and Triezenberg, S.J. (2006) Physical and functional interactions of Arabidopsis ADA2 transcriptional coactivator proteins with the acetyltransferase GCN5 and with the cold-induced transcription factor CBF1. Biochim. Biophys. Acta, 1759, 69-79.

Millar, C.B. and Grunstein, M. (2006) Genome-wide patterns of histone modifications in yeast. Nat. Rev. Mol. Cell Biol. 7, 657-666.

Mujtaba, S., Zeng, L. and Zhou, M.-M. (2007) Structure and acetyllysine recognition of the bromodomain. Oncogene, 26, 55215527.

Nourani, A., Utley, R.T., Allard, S. and Côté, J. (2004) Recruitment of the NuA4 complex poises the PHO5 promoter for chromatin remodeling and activation. EMBO J. 23, 2597-2607.

Pandey, R., Müller, A., Napoli, C.A., Selinger, D.A., Pikaard, C.S. Richards, E.J., Bender, J., Mount, D.W. and Jorgensen, R.A. (2002) Analysis of histone acetyltransferase and histone deacetylase families of Arabidopsis thaliana suggests functional diversification of chromatin modification among multicellular eukaryotes. Nucleic Acids Res. 30, 5036-5055.

Pray-Grant, M.G., Daniel, J.A., Schieltz, D., Yates, J.R., III and Grant, P.A. (2005) Chd1 chromodomain links histone H3 methylation with SAGA- and SLIK-dependent acetylation. Nature, 433, 434438.

Recht, J., Tsubota, T., Tanny, J.C. et al. (2006) Histone chaperone Asf1 is required for histone $\mathrm{H} 3$ lysine 56 acetylation, a modification associated with $\mathrm{S}$ phase in mitosis and meiosis. Proc. Nat Acad. Sci. USA, 103, 6988-6993.

Robert, F., Pokholok, D.K., Hannett, N.M., Rinaldi, N.J., Chandy, M., Rolfe, A., Workman, J.L., Gifford, D.K. and Young, R.A. (2004) Global position and recruitment of HATs and HDACs in the yeast genome. Mol. Cell, 16, 199-209.

Samson, F., Brunaud, V., Duchêne, S., De Oliveira, Y., Caboche, M., Lecharny, A. and Aubourg, S. (2004) FLAGdb++: a database for the functional analysis of the Arabidopsis genome. Nucleic Acids Res. 32, D347-D350.

Sasaki, Y., Sone, T., Yoshida, S., Yahata, K., Hotta, J., Chesnut, J.D. Honda, T. and Imamoto, F. (2004) Evidence for high specificity and efficiency of multiple recombination signals in mixed DNA cloning by the multisite Gateway system. J. Biotechnol. 107, 233243

Tepperman, J.M., Zhu, T., Chang, H.-S., Wang, X. and Quail, P.H. (2001) Multiple transcription-factor genes are early targets of phytochrome A signaling. Proc. Natl Acad. Sci. USA, 98, 94379442.

Tepperman, J.M., Hudson, M.E., Khanna, R., Zhu, T., Chang, S.H. Wang, X. and Quail, P.H. (2004) Expression profiling of phyB mutant demonstrates substantial contribution of other phytochromes to red-light-regulated gene expression during seedling de-etiolation. Plant J. 38, 725-739.

Turck, F., Roudier, F., Farrona, S., Martin-Magniette, M.-L., Guillaume, E., Buisine, N., Gagnot, S., Martienssen, R.A., Coupland G. and Colot, V. (2007) Arabidopsis TFL2/LHP1 specifically associates with genes marked by trimethylation of histone $\mathrm{H} 3$ lysine 27. PLoS Genet. 3, e86, 0855-0866.

Vlachonasios, K.E., Thomashow, M.F. and Triezenberg, S.J. (2003) Disruption mutations of $A D A 2 b$ and $G C N 5$ transcriptional adaptor genes dramatically affect Arabidopsis growth, development, and gene expression. Plant Cell, 15, 626-638

Yamada, K., Lim, J., Dale, J.M. et al. (2003) Empirical analysis of transcriptional activity in the Arabidopsis genome. Science, 302 842-846.

Yamauchi, T., Yamauchi, J., Kuwata, T., Tamura, T., Yamashita, T., Bae, N., Westphal, H., Ozato, K. and Nakatani, Y. (2000) Distinct but overlapping roles of histone acetylase PCAF and of the closely related PCAF-B/GCN5 in mouse embryogenesis. Proc. Natl Acad. Sci. USA, 97, 11303-11306.

Zhang, X., Yazaki, J., Sundaresan, A. et al. (2006) Genome-wide high-resolution mapping and functional analysis of DNA methylation in Arabidopsis. Cell, 126, 1189-1201. 